\title{
Breath volatile compounds and conjugated polyunsaturated fatty acids as metabolic biomarkers reflecting the interaction between chitin-glucan and the gut microbiota.
}

\author{
Julie Rodriguez $^{1}$, Audrey M. Neyrinck ${ }^{1}$, Julie-anne Nazare ${ }^{2}$, Zhengxiao Zhang ${ }^{3}$, \\ Benjamin Seethaler ${ }^{4}$, Candido Robles Sanchez ${ }^{1}$, Giulio Muccioli ${ }^{5}$, Véronique Maquet ${ }^{6}$, \\ Stephan C. Bischoff ${ }^{4}$, Jens Walter ${ }^{7}$, Martine Laville ${ }^{2}$ and Nathalie M. Delzenne ${ }^{1}$ \\ ${ }^{1}$ Metabolism and Nutrition Research Group, Louvain Drug Research Institute, Université catholique de Louvain, \\ Brussels, Belgium, \\ ${ }^{2}$ Rhône-Alpes Research Center for Human Nutrition, Lyon, France, \\ ${ }^{3}$ Department of Medicine, University of Alberta, Alberta, Canada, \\ ${ }^{4}$ Institute of Nutritional Medicine, University of Hohenheim, Stuttgart, Germany, \\ ${ }^{5}$ Bioanalysis and Pharmacology of Bioactive Lipids Research Group, Louvain Drug Research Institute, Université \\ catholique de Louvain, Brussels, Belgium, \\ ${ }^{6}$ KitoZyme SA, Parc industriel des Haut-Sarts, Herstal, Belgium and \\ ${ }^{7}$ Department of Agricultural, Food \& Nutritional Science and Department of Biological Sciences University of Alberta, \\ Alberta, Canada
}

\begin{abstract}
Introduction: Dietary fibers (DF) are considered as beneficial nutrients for health. Current data suggest that their interaction with the gut microbiota largely contributes to their physiological effects. The FiberTAG project ${ }^{(1)}$ innovates in searching for new biomarkers reflecting the health effect of dietary fibers, including chitin-glucan (CG, extracted from the fungal exoskeleton Aspergillus niger). CG improves metabolic disorders associated with obesity in mice, but its effect on gut microbiota composition and function has never been evaluated in vivo in humans.

Materials and Methods: CG (KitoZyme, Belgium) was given to healthy volunteers $(\mathrm{n}=15)$, during three weeks $(4.5 \mathrm{~g} / \mathrm{day})$. Volatile Organic Compound (VOC) metabolites released in breath were analyzed using SYFT methodology. Fatty acid (FA) profiling was assessed in stool samples, by gas-liquid chromatography ${ }^{(2)}$. The gut microbiome was analyzed by Illumina sequencing (V5-V6 region of $16 \mathrm{~S}$ rRNA).

Results: Three weeks of CG supplementation was well tolerated and lead to changes in the kinetics of breath VOC, especially the short-chain fatty acid, alcohols and alkanes. Fecal vaccenic acid, produced upon the bacterial metabolism of fatty acids, was significantly increased by CG. Several bacterial genera were correlated with breath VOC (i.e. 2 methylbutyric acid and RuminococcaceaeUCG005). Moreover, Roseburia, often presented as a butyrate producer, was positively correlated with the production of a rumenic acid isomer "cis-9,cis-11-18:2".

Discussion: We show that breath VOC analysis, a non-invasive methodology, reveals characteristics of microbiota-CG interactions. We also show that CG selectively changes the profile of FA metabolites, in favor of vaccenic acid, another bioactive metabolite produced by Roseburia, prone to act on host physiology. This study will help to establish a set of new biomarkers linking insoluble DF and gut microbiota, with focus on their interest in human health.
\end{abstract}

\section{Acknowledgements}

JR and AMN contributed equally to this work

\section{Conflict of Interest}

There is no conflic of interest

\section{References}

1. FiberTAG project from European Joint Programming Initiative "A Healthy Diet for a Healthy Life" https://www.fibertag.eul

2. Druart et al. (2014) Lipids Apr 49(4), 397-402. 